\title{
CASE SERIES OF INTRACANAL REHABILITATION WITH LIGHT TRANSMITTING POSTS
}

Anjali S. Vats ${ }^{1}$, Ankur Vats ${ }^{2}$, Paras Kothari ${ }^{3}$

${ }^{1}$ Sr.Lecturer in the Department Of Conservative Dentistry \& Endodontics, Bhojia Dental College and Hospital, Baddi, Himachal Pradesh. ${ }^{2}$ Sr.Lecturer in the Department Of Conservative Dentistry \& Endodontics, Bhojia Dental College and Hospital, Baddi, Himachal Pradesh ${ }^{3}$ Ex-PG student K.M. Shah Dental College \& Hospital, Vadodara, Gujarat, India.

\begin{abstract}
Corresponding Author: Anjali S. Vats Mobile: +91-9876064652 E-mail address: rootsandcrown@yahoo.com

Received: $12^{\text {th }}$ Dec. 2013 Accepted: $2^{\text {nd }}$ Feb. 2014 Online: $25^{\text {th }}$ May 2014

\section{INTRODUCTION}

The functional and esthetic rehabilitation of compromised, root filled teeth presents a difficult restorative problem for the practicing dentist. Many anterior teeth that require post retained restorations are severely weakened as a result of recurrent caries extending in to the radicular dentin, around pre-existing posts or the fact that the pulp has become necrotic prior to the completion of the root formation in a young patient. Other, less common conditions include developmental anomalies such as fusion and gemination, internal resorption, and iatrogenic damage resulting in large access preparations. $^{1}$

Loss of tooth structure in these affected teeth may result in little or no remaining crown structure and weakened thin walled roots with widely flared canals. Such canal geometry presents a risk for root fracture and very wide, tapered, and unretentive post. Resistance to fracture of root is directly related to the thickness of the remaining dentin, especially in the bucco-lingual direction. Excessive flaring during endodontic treatment or over preparation of the canal space for a post can increase the risk of failure. ${ }^{2}$ Therefore the need to preserve and protect the
\end{abstract}

ABSTRACT

Intra-radicular rehabilitation is a procedure in which the root canals with very little remaining dentin thickness are strengthened by the use of such material that bonds and reinforces the root structure. In this case report, intra radicular rehabilitation with the use of seventh generation bonding agent and flowable composite is described and discussed as a treatment approach for the restoration of a thin fragile root. The procedure provides for an extended clinical application of the all-in-one bonding agent resulting in conservative, reliable treatment approach for the treatment of compromised root canals due to variety of reasons

Keywords: Bonding agent, Post and core technique, Tooth fracture

remaining dentin for maximum root strength is equally important as retention. In addition, a sufficient buccal dentinal wall of root must be conserved in maxillary anterior teeth because of its function as a fulcrum towards horizontally directed forces. ${ }^{3}$

In 1987, Lui introduced a reinforcement technique involving the reconstitution of lost intraradicular dentin in conjugation with the creation of a size matched post canal of predetermined length to support a functional post crown. It involved acid etching of the internal radicular dentin in combination with adhesive bonding and lining of the thin canal wall with autocuring resin composite. Besides reinforcement it also reconstituted a new post canal space that could accommodate a size matched, passive, parallel sided, well-fitting post. ${ }^{4}$

This technique re-established the continued serviceability of badly damaged non vital teeth that were previously deemed un-restorable and consequently condemned for extraction. These following case reports illustrate the enhancement of post crown retention in composite reconstituted, flared canals of compromised teeth. 


\section{CASE 1:}

Case History: A 25 year old female patient reported to the Department of Conservative dentistry and Endodontics with the complaint of broken upper anterior tooth. History revealed a fall during her childhood. Medical history was non-contributory. On intraoral examination Ellis Class III fracture with 21 and Ellis Class II fracture with 11 was noticed (Figure 1). ${ }^{21}$ was discoloured, exhibited grade I mobility, non-tender to percussion and palpation, with deep bite and normal overjet. Electric pulp testing gave negative response. Radiograph revealed an oblique fracture of the crown involving the pulp space and periapical radiolucency. Initial treatment plan included the root canal therapy followed by post and core and final restoration with porcelain fused to metal crown. Whereas 11 responded normal to the electric pulp vitality test therefore a composite build up was done for it.

Root canal therapy was initiated and access cavity prepared in 21. Working length was determined and biomechanical preparation was done to ISO size 70 with the K-Files (Dentsply Maillefer, Ballalgues, Sweden). The tooth was obturated with laterally condensed gutta-percha technique (Hygenic, Coltene/Whaledent, OH, USA). To prepare the post space, gutta-percha was removed leaving approximately $5 \mathrm{~mm}$ apically. Post space preparation was completed using Peeso reamers (Mani inc, Japan). A radiograph was taken in which excessive loss of dentin in the coronal 3rd of the root canal was observed. A decision was made to reinforce the root and at the same time block the gouged portion with composite resin. The post space was modified in the following manner. The post space was irrigated with $3 \%$ sodium hypochlorite and EDTA 17\% (Dent wash, Prime Dental, Mumbai, India) to remove the smear layer. A light emitting post Luxapost (DMG, Germany) of No.3 size was selected after try in as it was found to allow sufficient amount of composite material all around. Prime and Bond NT (Dentsply, Caulk) a seventh generation, all in one bonding agent was applied to the dentinal walls and light cured following manufacturer's instructions. Tetric flow (Ivoclar Vivadent AG, Bendererstrasse, Liechtenstein) flowable composite resin was injected in to the canal. The selected light emitting post was inserted into the canal to the full depth to ensure desired post length maintaining the central position to allow for the uniform thickness of composite all around. Excess material was removed and the material was cured for 60 seconds by holding the light cure gun in contact with the light emitting post
(Figure 2). After curing, core build up was done using Fluorocore 2 (Dentsply Caulk). Tooth preparation for PFM crown was done (Figure 3). A rubber base impression after gingival retraction was taken and tooth was temporized. Finally a porcelain fused to metal crown was cemented using GIC cement (Figure 4).

\section{CASE 2:}

Case History: A 28 year old female patient reported to the Department of Conservative dentistry and Endodontics with the complaint of broken upper anterior tooth. History revealed a fall during her childhood. Medical history was non-contributory. On intraoral examination Ellis Class III fracture with 21 was noticed (Figure 5) ${ }^{21}$ was discoloured, exhibited grade I mobility, non-tender to percussion and palpation, with deep bite and increased overjet. Electric pulp testing gave negative response with respect to 21 and 22. Radiograph revealed an oblique fracture of the crown involving the pulp space and a periapical radiolucency. Initial treatment plan included the root canal therapy followed by post and core and final restoration with porcelain fused to metal crown with respect to 21 and 22. However, the patient due to financial reasons did not agree for PFM crowns. Treatment plan was changed. After root canal treatment for 21 and 22, whereas non-vital bleaching with Insideout technique followed by Post placement for 21 was planned.

Root canal therapy was initiated, access cavity was prepared. Working length was determined and biomechanical preparation was done to ISO size 80 with the K-Files with respect to 21 and with K-file ISO size 45 with respect to 22 (Dentsply Maillefer, Ballalgues, Sweden). The teeth were obturated with laterally condensed gutta-percha technique (Hygenic, Coltene/Whaledent, $\mathrm{OH}, \mathrm{USA}$ ). Inside-out in-office bleaching was done in 21 using Pola Office (SDI, Australia) till desirable shade was achieved (Figure 6). Then to prepare the post space with respect to 21, guttapercha was removed leaving approximately $5 \mathrm{~mm}$ apically. Post space preparation was completed using Peeso reamers (Mani inc, Japan). A radiograph was taken in which excessive loss of dentin in the coronal $3^{\text {rd }}$ of the root canal was observed.

A decision was made to reinforce the root and at the same time block the gouged portion with composite resin. The post space was modified in the following manner. The post space was irrigated with $3 \%$ sodium hypochlorite and EDTA 17\% (Dent wash, Prime Dental, Mumbai, India) to remove the smear layer. A light emitting post Luxapost (DMG, Germany) of No.3 
size was selected after try in as it was found to allow sufficient amount of composite material all around. Prime and Bond NT (Dentsply, Caulk) a seventh generation, all in one bonding agent was applied to the dentinal walls and light cured following manufacturer's instructions. Tetric flow (Ivoclar Vivadent AG, Bendererstrasse, Liechtenstein) flowable composite resin was injected in to the canal. The selected light emitting post was inserted into the canal to the full depth to ensure desired post length maintaining the central position to allow for the uniform thickness of composite all around. Excess material was removed and the material was cured for 60 seconds by holding the light cure gun in contact with the light emitting post (Figure 7). After curing, core build up was done using Tetric Ceram (Ivoclar Vivadent AG, Bendererstrasse, Liechtenstein) (Figure 8).

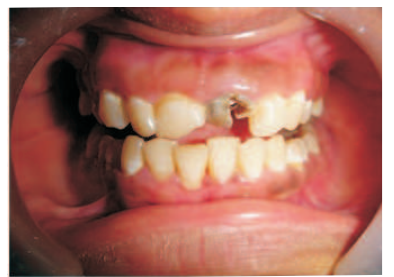

Figure 1:

Pre-operative

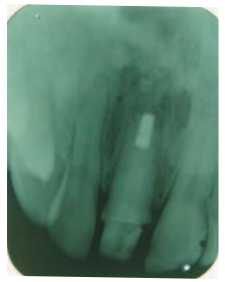

Figure 3: IOPA after Post cementation and core build up

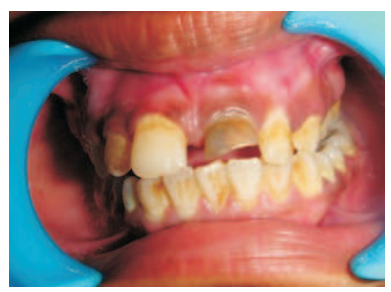

Figure 5: Pre-operative

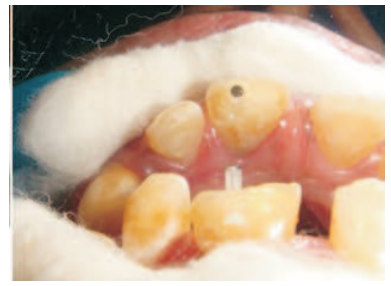

Figure 7:

Post cementation

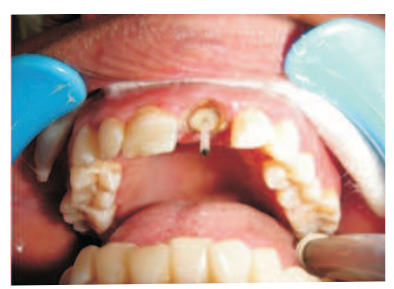

Figure 2:

Light Transmitting Post Cement

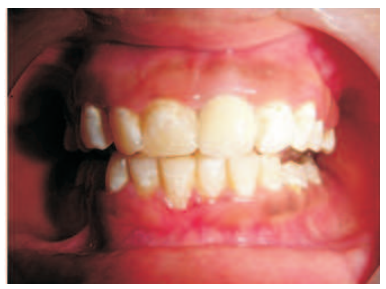

Figure 4:

Post-operative

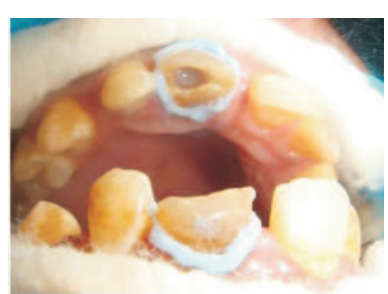

Figure 6: Inside out In-office Bleaching

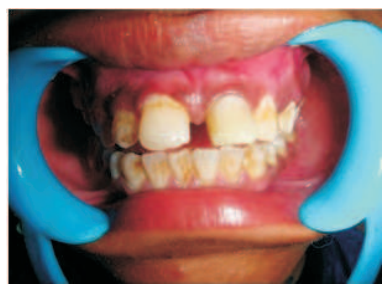

Figure 8:

Post-operative

\section{DISCUSSION:}

Restoration of the endodontically treated teeth with a dowel and core before placement of the final restoration is a common procedure and various techniques have been recommended and described in the literature.

These particular cases presented an uniqueness in problem that the root walls were very thin, restoring the same with the traditional method would have resulted in the chance of fracture of root, alternatively, restoration with the pre-fabricated post would have resulted in obturation of the large defect with the cementing medium, creating a very weak area in the entire post-core-crown-tooth complex. In 2004, Anil Kishen et al., suggested that the structure of inner dentin, which surrounds the root canal is less mineralized and has more collagen, hence possess low modulus of elasticity. ${ }^{6}$ It is proposed in the literature that at least $1 \mathrm{~mm}$ of dentin be maintained around the entire circumference of the channel to prevent root fracture. $^{7}$

The anterior teeth protect the posterior teeth from lateral forces through anterior guidance. In cases with very steep anterior guidance or deep vertical overlap like in this case, maxillary anterior teeth are subjected to horizontal protrusive and lateral forces from the mandibular anterior teeth. The loss of tooth structure destroys the structural integrity allowing the greater flexion of the tooth under function and restorations for damaged anterior teeth with heavy function are designed to resist flexion. Restorative components should be stronger than would be required for teeth with edge to edge relationship and vertical forces.

Retention of posts in these thin, flared canals traditionally has been achieved with custom cast post and cores that reflect the internal canal shape, but this technique risks failure from root fracture. Composite resin coupled with a central metal post can be valuable in reinforcing internal radicular walls of roots thinned by caries or other dentin loss. The resultant dentin/composite post complex is upto $50 \%$ stronger than roots restored conventionally. ${ }^{8}$ A metal post cemented into the composite matrix, combine the cervical stiffening benefit of a rigid post with protective elasticity of the composite matrix. In an in-vitro study of damaged teeth with flared canals and thin dentinal walls, metal posts were significantly stronger than fiber posts; however, the mode of failure of fiber posts was protective against fracture. ${ }^{9}$ The Finite element analysis of different post systems had shown maximum stress in the coronal and middle third of the root. Maximum stress was seen on the inner dentinal wall in case of stainless steel post followed by cast gold and 
carbon fiber post, both in the models without reinforcement as well as in the reinforced models. ${ }^{10}$

Use of auto curing composite and light curing composite resins in the conventional manner can give rise to problems in the depth of the root canal due to inaccessibility.

The use of light transmitting posts in conjugation with light curing composite resins eliminates the difficulty associated with a rapidly polymerizing auto-curing composite resin. It ensures complete polymerization of the light curing composite resin. In fact the curing is so complete that Tjan et al., found that the retentive value of the composite reinforcement bonded to the root canal wall is significantly higher than that of a custom cast core luted to the root canal wall with zinc phosphate cement. ${ }^{11}$ In the composite lining and reinforcement technique, the intraradicular composite on polymerization adapts very accurately to the parallel sided post thus establishing a patent post canal that accurately matches its intended post. Because posts are intended to provide retention and resistance to displacement of the core, the post canal should be as small as the apical dentin portion of the root canal, would fit the smallest acceptable size post. ${ }^{4}$ The use of the matching size, grooved burnout casting post also contributes to overall retention. The cast post and core results in a passive fit thus avoiding the stresses due to polymerization shrinkage that might occur with the use of dual cure resin cement and prefabricated post. With the availability of low viscosity flowable composites, the packing of the composite into the root canals has become easier and aid in obtaining uniform thickness of the composite all around. The introduction of $7^{\text {th }}$ generation bonding agent Prime N Bond NT (Dentsply), the bonding procedure is further simplified. There is some concern regarding coronal microleakage in endodontically treated teeth because neither gutta-percha nor cemented prefabricated and custom posts and cores can effectively prevent the crown down leakage from the oral environment. ${ }^{12-17}$ The removal of the smear layer and the adhesion of resin materials to the root canal wall have been shown to decrease the leakage. ${ }^{18-20}$

\section{CONCLUSION}

The availability of the light transmitting plastic posts and developments in dentin bonding systems and composite resins would go a long way in enabling the dentist to try newer techniques in keeping with the conservative rehabilitation for the restoration of badly damaged teeth.

\section{REFERENCES:}

1. Lui JL. Composite resin reinforcement of flared canals using light transmitting plastic posts. Quintessence Int 1994;25:31319.

2. Stockton LW. Fracture affecting the retention of post systems: A literature review. J Prosthet Dent 1999;81(4):380-82.

3. Tjan AHL, Whang SB. Resistance to root fracture of dowel channels with various thickness of buccal dentin walls. J Prosthet Dent 1985;53(4):496-500.

4. Lui JL. A technique to reinforce weakened roots with post canals. Endod Dent Traumatol 1987;3:310-14.

5. Lui JL. Composite resin reinforcement of flared canals using light transmitting plastic posts. Quintessence Int 1994;25:31319.

6. Kishen A, Kumar GV, Chen NN. Stress-strain response in human dentin: Rethinking fracture predilection in post core restored teeth. Dent Traumatol 2004;20:90-100.

7. Standlee JP, Caputo AA, Hanson EC. Retention of endodontic dowels: Effect of cement, dowel length, diameter and design. J Prosthet Dent 1978;39:400-5.

8. Saupe WA, Glaskin AH, Radke RA Jr. A comparative study of fracture resistance between morphological dowel and cores and a resin-reinforced dowel system in the intraradicular restoration of structurally compromised roots. Quintessence Int 1996;27:483-89.

9. Newman MP, Yaman P, Dennison J, Rafter M, Billy E. Fracture resistance of endodontically treated teeth restored with composite posts. J Prosthet Dent 2003;89:360.

10. Kaur A, Meena N, Shubhashini N, Kumari A, Shetty A. A comparative study of intra canal stress pattern in endodontically treated teeth with average sized canal diameter and reinforced wide canals with three different post systems using finite element analysis. J Conserv Dent 2010;13:28-33.

11. Tjan AHL, Whang SB. Resistance to root fracture of dowel channels with various thickness of buccal dentin walls. J Prosthet Dent 1985;53(4):496-500.

12. Swanson KS, Madison S. An evaluation of coronal microleakage in endodontically treated teeth. Part I. Time periods. J Endod 1987;13:56-59.

13. Madison S, Swanson KS, Chiles S. An evaluation of coronal microleakage in endodontically treated teeth. Part II. Sealer types. J Endod 1987;13:109-12.

14. Madison S, Wilcox L. An evaluation of coronal micro leak-age in endodontically treated teeth. Part III. In vivo study. J Endod 1988; $14: 455-58$.

15. Torabinejad M, Ung B, Kettering JD. In vitro bacterial penetration of coronally unsealed endodontically treated teeth. J Endod 1990;16:566-69.

16. Magura ME, Kafrawy AH, Brawn CE Jr, Newton CW. Human saliva microleakage in obturated root canals: An in vitro study. J Endod 1991; 17:324-31.

17. Freeman MA, Nicholls JI, Kydd WL, Harrington GW. Leakage associated with load fatigue-induced preliminary failure by full crowns placed over post and core systems. J Endod 1998;24:26-52.

18. Taylor JK, Jeansonne BG, Lemon RR. Coronal leakage: Effects of smear layer, obturation technique and sealer. J Endod 1997;23:508-12.

19. Wennberg A, Ostravik D. Adhesion of root canal sealers to bovine dentin and gutta-percha. Int Endod J 1990;23:13-19.

20. Tjan AHL, Grant BE, Dunn JR. Microleakage of composite resin cores treated with various dentin bonding agents. J Prosthet Dent 1991;66:24-29. 